\title{
Sobre el encierro de los pobres en los tiempos modernos
}

\author{
Juan Manuel Santana Pérez ${ }^{*}$
}

Durante los tiempos modernos, especialmente durante los siglos XVII y XVIII, se produce una mayor preocupación del poder con respecto a los pobres (reflejada de paso en el aumento de la documentación), produciéndose en consecuencia una elevación cuantitativa del internamiento con el incremento paralelo de la capacidad hospitalaria ${ }^{1}$. Se recoge a mendigos, vagabundos y marginales en general con la intención de recluirlos en las instituciones de beneficencia existentes?

El internamiento habria que entenderlo como expresión directamente ejercida del poder de las clases dominantes, ya que sus intenciones variarán en función de las necesidades socio-económicas. En ambas vertientes, represiva o de ayuda, la beneficencia sería detentada por la cúspide social ${ }^{3}$.

Las discusiones entre los historiadores sobre la reforma de la beneficencia en las ciudades europeas, ha dejado sin dar la importancia que se merece al problema de la nueva situación social, que estuvo en el origen

\footnotetext{
Universidad de Las Paimas de Gran Canaria.

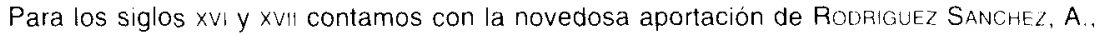
"La historia de la violencia: espacios y formas en los siglos XVI y Xv\|l" en BAhro, C. (ed.), Historia a Debate. Santiago de Compostela, 1995, t. Il. págs. 117-127.

Para un estado de la cuestıón sobre el particular nos remitimos a dossier de Historia Social, n." 13, primavera-verano 1992; concretamente, sobre el periodo que nos interesa, están los artículos siguientes, CARASA SOTO, P., "La historia de los pobres: de las bienaventuranzas a la marginación", págs. 77-99; y DIEZ, F., “Estructura social y sistema benéfico-asistencial en la ciudad preindustrial", págs. 101-121. También en MOREno MaRTINEZ, D., y BETRAN, J.L., "Justicia criminal en la Cataluña moderna: Estudios y perspectivas de investigación», en BARRO, C. (Ed.), Historia a Debate. Santiago de Compostela, 1995, t. II, pags. 103-115. Sobre la inserción de este tema en la moderna historiografía, contamos con el prólogo de C. Martínez-Shaw en SANTANA PEREZ, J. M. y Monzon Pefdomo. M. E., Hospitales de La Laguna durante el siglo xvili. La Laguna. Ayuntamiento de San Cristóbal de La Laguna, 1996, págs. 7-9.

Terradillos, J., "Un supuesto de estado peligroso: la vagancia habitual», Materiales, n." 10. Barcelona, julio-agosto 1978, pág. 81.
} 
de este movimiento y que obligó a las autoridades urbanas a buscar nuevas soluciones ${ }^{4}$.

Los hospitales y hospicios absorberán a quienes vivan en estado de indigencia, evitando con ello la agudización del conflicto social. Debemos pensar que estas masas desocupadas estaban prestas a apoyar cualquier revuelta para obtener mejoras en su nivel de vida, como sucedió en muchos lugares de Europa. Pese a no ser los sujetos directos de los motines fueron utilizados como fuerza de choque y, lo que es más importante, los grupos dominantes los temían; llegaron a formar una subcultura popular, con un lenguaje particular cuidadosamente diseñado para excluir al resto ${ }^{5}$.

A lo largo del siglo xIx irá desapareciendo progresivamente la función penal de los establecimientos benéficos, que acrecentarán su objetivo asistencial, destinándose otro tipo de instituciones al otro fin de forma específica.

En los momentos de coyunturas económicas menos adversas, la reclusión sigue teniendo importancia para el Estado. A la labor represiva se le añade la posibilidad de dar empleo a quienes habian sido encerrados con anterioridad. Es decir que el derecho del Estado a castigar, se transforma en derecho a prevenir. El internamiento como manifestación de una política general responde en la Monarquía española a la crisis económica del siglo xVII y, parcialmente, del xVIII, mediante la detención o la dedicación coactiva al trabajo del indigente ${ }^{6}$. En España la instrumentalización de la ley al servicio del sistema de relaciones sociales aparece recubierta del correspondiente sistema de valores de orden moral?

Las ayudas del Estado a los sectores desposeidos en situaciones económicamente dificiles, evitaban que el pauperismo sobrepasara los niveles mínimos de supervivencia. La articulación de un aparato benéfico era una necesidad para el mantenimiento y reproducción del sistema porque en esas situaciones concretas, con unas determinadas relaciones sociales de producción y una estructura política concreta, se hacía obligatoria la

\footnotetext{
GFMEREK, B., "La población marginal entre el Medievo y la era moderna", en Agricultura y desarrollo del capitalismo. Madrid, Comunicación, 1974, pág. 256.

Bunke, P. La cuitura popular en la Europa moderna. Madrid, Alianza Universidad. 1991. pág. 91

Los aspectos relacionados con la atenuación de las contradicciones sociales en España están estudiados en FLYNN, M. Sacred charity confraternities and social welfare in Spain. 14001700, L.ondres, Macmillan, 1989 23-35

Terradilios Basoco, J., Peligrosidad social y Estado de Derecho. Madrid, Akal, 1981, págs.
} 
existencia de marginalidad y por consiguiente de las instituciones de beneficencia. Somos partícipes del pensamiento marxiano en que:

“... tanto las relaciones juridicas como las formas de Estado no pueden comprenderse por si mismas ni por la llamada evolución general del espiritu humano, sino que radican por el contrario, en las condiciones materiales de vida cuyo conjunto resume Hegel, siguiendo el precedente de los ingleses y franceses del siglo xvirr, bajo el nombre de sociedad civil, y que en la anatomia de la sociedad civil hay que buscarla en la Economía Política".

Aunque el pobre generalmente era victima de las circunstancias naturales, no se podia permitir que llevase una forma de vida errante con costumbres mal vistas socialmente. El internamiento evitará sus actividades a la vez que justificará la mediación por parte del Estado como responsable máximo del sistema económico. Fue precisamente en el momento en que la idea de la pobreza se secularizó cuando los pobres quedaron a cargo del Estado ${ }^{9}$. Se pretendía acabar con la miseria poniendo fuera de la dinámica social y manteniendo con "la caridad" a quienes no contasen con los suficientes recursos económicos.

Con la lógica perspectiva histórica que nos proporciona el tiempo, podemos afirmar que no estuvo en la beneficencia la salida adecuada a la marginalidad y los conflictos sociales derivados o agudizados por ésta, pero constituyó una pieza importante a la hora de atenuar las consecuencias de las contradicciones socio-económicas imperantes.

La gran cantidad de limosnas que daban las clases acomodadas, el clero, la nobleza y algunos sectores de la burguesia, es lo que ha inducido a hablar del paternalismo de las clases dominantes, además de las comidas repartidas en las puertas de los conventos o de alguna gran casa, junto con la existencia de cofradias aparentemente movidas por el filantropismo humanitario, pero nunca se consiguió sofocar el hambre y acabar con la mendicidad.

Al poder estatal no le era posible el control absoluto de la situación, pero era el único sujeto con verdaderas posibilidades de lograr el exterminio de esa "plaga social» y capaz de recaudar, organizar y centralizar las

Marzx. K., "Prólogo de la Contribución de la Economia Politica", en Obras Escogidas de Marx y Engels. Madrid. Fundamentos, 1977, págs. 372-373.

Esto ha sido demostrado para Inglaterra por HIMmelfarlo, G., La idea de la pobreza. México, F.C.E., 1988, pág. 12. Nosotros lo hemos abordado para la Monarquia española. con su plasmación en el caso canario en Santana PEREz, J.M. Cunas de Expósitos y Hospicios en Canarias (1700-1837). Las Palmas de Gran Canaria. Cabildo Insular de Gran Canaria. 1994. 
limosnas de todas las clases sociales potencialmente donantes con unos intereses convergentes.

Parece estar claro que durante el siglo XVIII fue en el reinado de Carlos III cuando realmente se formó en España un verdadero plan de asistencia pública. Las disposiciones que se dictaron en su reinado para el ejercicio de la caridad constituyeron un sistema general de beneficencia que perseguía como fin formal: desterrar la vagancia y la mendicidad "voluntaria».

Gaspar Melchor de Jovellanos en sus elogios al monarca señalaba que:

«... se ganó el amor y gratitud del pueblo por sus providencias y establecimientos de este benéfico soberano..." ".

Le da el adjetivo de benéfico a la vez que resalta como uno de los elementos principales de su reinado, las empresas de beneficencia acometidas.

A finales de dicho reinado en España había 773 hospitales que incluian:

$\begin{array}{lr}\text { Capellanes } & 609 \\ \text { Empleados } & 1.105 \\ \text { Facultativos } & 1.382 \\ \text { Sirvientes } & 2.477 \\ \text { "Enfermos" } & 7.699 \\ \text { "Enfermas» } & 3.283 \\ \text { Locos } & 359 \\ \text { Locas } & 336 \\ \text { Expósitos } & 1.612 \\ \text { Expósitas } & 763\end{array}$

No obstante, habia 51 cunas de expósitos con 173 empleados para cuidar a 1.918 niños y 2.048 niñas.

Además funcionaban 88 hospicios que englobaban a:

$\begin{array}{lr}\text { Capellanes } & 71 \\ \text { Empleados } & 392 \\ \text { Sirvientes } & 153 \\ \text { Hombres } & 3.831 \\ \text { Mujeres } & 4.937 \\ \text { Niños } & 1.926 \\ \text { Niñas } & 1.853\end{array}$

De Jovellanos, G.M., "Elogio de Carlos III", en Caso Gonzalez, J., Obras en prosa. Madrid. Castalia, 1976, pág. 177. 
A esto se le unian otras 26 instituciones que respondian al título de "Casas de Reclusión", que solamente acogian a:

$\begin{array}{ll}\text { Empleados } & 124 \\ \text { Reclusos } & 413 \\ \text { Reclusas } & 658\end{array}$

FUENTE: British Library. The Department of Manuscripts, Fondo Egerton, Legajo 376 , fol. 19 recto.

El gobierno central puso énfasis en la beneficencia para atenuar las contradicciones sociales agudizadas por las necesidades económicas. En el noroeste de Europa y las áreas más desarrolladas de la Península Ibérica, durante los siglos XVII y XVIII paralelamente al surgimiento de las manufacturas, se asiste a las viejas formas de castigo corporal y al nacimiento de nuevas instituciones (principalmente los hospicios) dentro de las cuales se impondrán las formas de la disciplina proletaria, siendo obligadas esas personas al aprendizaje para hacer posible la existencia misma de la sociedad burguesa".

A medida que aumentan los grupos marginales, el aparato de Estado se va responsabilizando más de su asistencia. Como hemos apuntado, en ocasiones también cumplió un papel positivo para los pobres. De necho, todas las personas que creyeron tener derecho a la beneficencia pidieron asistencia. Los ciegos, los sordomudos, los veteranos mutilados de guerra pedian ayuda. La beneficencia empezó cada vez más a servir a un proyecto dual, es decir, asistencia a los necesitados y recompensa a los patriotas heridos ${ }^{12}$.

En los hospitales eran internadas anualmente una gran cantidad de población de ambos sexos. Ya entrado el siglo XVIII, la orfandad se traspasará al sector público, a medida que crece este problema.

En España, la acción de los ilustrados sobre la vieja herencia de la caridad estamental tiene mucha importancia. No sólo por la decisiva aportación que hacen a su época sino por el recuerdo de viejas intentonas renacentistas frustradas y por el anticipo de muchas soluciones que adoptarán los liberales ${ }^{13}$.

Pavarini, M., Control y dominación. México, Siglo Veintiuno, 1983, págs. 152-153.

ROSFN, G., "Hospitals, medical care and social Policy in the French Revolution". Bulletin History Medical, n. 30, Londres, 1956, págs. 135-140

'.. Carasa Soto, P., Crisis del Antiguo Régimen y acción social en Castilla. Madrid, Junta de Castilla y León, 1988. pág. 121 
Era precisa una cohesión ideológica que justificase este hecho y a lo largo de la centuria se irá extendiendo el pensamiento de que el desarrollo de la beneficencia es un deber moral de los gobernantes y del Monarca como su máximo representante. Apreciamos la aparición entre las instituciones de la Monarquia Absoluta, la idea de la clase burguesa de que la virtud es un asunto de Estado, el cual debe dictar una normativa para imponerla y establecer una autoridad para que sea respetada perpetuamente, claras características de modernidad.

Al concluir el siglo XVIII, el Estado moderno tenía a su cargo la parte más importante de la beneficencia, aunque todavia existian ayudas y colaboraciones de tipo particular en la asistencia, manifestadas tanto a nivel organizativo como en la caridad personal de miembros del nivel más alto de la sociedad. Sin embargo, la Corona en todo momento tenía en cuenta su deber y lo ejecutaba de muy diversas formas.

Pero también hubo detractores del sistema secularizado de hospicios. Personajes tan poco sospechosos como Jovellanos dijeron que contra la práctica la razón aconseja la inconveniencia de los hospicios ya que ni la higiene ni la salud, ni la moral, ni la economía permitirian sostener esas instalaciones ${ }^{14}$. Aunque creemos con Callahan que a pesar de la crítica contra los hospicios movida por !as nuevas corrientes intelectuales de la época, la oposición más intensa y tenaz al sistema de ayuda a los pobres que fue llevada a cabo por el Estado después de 1750 provino de un cierto silencio público ${ }^{15}$.

De esta amalgama de programas bien definidos sobre el aparato benéfico, salió la realidad de la política seguida por la Corona, aunque no se cumplió en su totalidad porque en muchos casos rozaba la utopía.

Sin embargo, debemos entender las estructuras del Estado como algo dialéctico, no como una serie de mecanismos ahistóricos y perfectos, sino como resultado de la coyuntura. Es decir, que la beneficencia en su vertiente represiva es impuesta por los grupos dominantes, pero en cuanto medida asistencial es demandada por los dominados. La composición resultante es inevitablemente contradictoria: nunca se da una solución totalmente libre de problemas al reto de las posiciones políticas ${ }^{16}$.

\footnotetext{
1.4 PfRez Esievt L, M.R., El problema de los vagos en la España diel siglo XVIII. Madrid, Confederación General de Cajas de Ahorros, 1976, pags. 328-329.

CALLAHAN. W. J., "The problem of confinement: An aspect of poor relief in EighteenthCentury Spain». Hispanic American Historical Review, LI, 1971, pág. 24.

ESFInG-ANDERSEN, G., FriedLAND, R. y Olin WRIGHT. E.. "Los modos de la lucha de clases y el Estado capitalista», en VV.AA., Capitalismo y Estado. Madrid, Revolución, 1985, págs. 103-104.
} 
Se hacía precisa la fundación de instituciones benéficas para quitar a los pobres de las calles. En todo momento fue reivindicada su importancia a la vez que se multiplican los acuerdos con respecto a dichos centros, entre los representantes de los poderes locales y del gobierno central.

Los hospitales durante el siglo XVIII, en su funcionamiento y en sus objetivos, no tienen relación sólo con aspectos médicos como sucede en la actualidad. Eran una instancia del orden, tanto monárquico como burgués o aristocrático, ya que coinciden los intereses, combinada con una actividad sanitaria, aunque de forma complementaria porque no era el objetivo principal.

Esas instituciones tenían una relación directa con el poder real, que los habia colocado bajo la autoridad del gobierno civil para hacerlos más eficientes. El propio poder comprende la importancia de centros de internamiento, los promociona no sólo en el terreno ideológico a través de una fuerte exaltación de su papel, sino incluso infraestructuralmente, lo cual redunda en un funcionamiento mejor que en etapas anteriores.

Durante el Trienio Liberal con el nuevo código penal, diversas penas serán castigadas con reclusión en casas de corrección. Para ello se envía un pequeño mandato de cuatro artículos, donde el segundo obligaba a que en cada provincia hubiese al menos una casa de corrección para hombres y otra para mujeres con dos departamentos - recogido en el tercer artículo- sin comunicación alguna entre sí, siendo uno de reclusión y otro de corrección ${ }^{17}$.

Por otro lado, como existía un interés por eliminar la beneficencia individual por su falta de organización con respecto a las medidas públicas, el gobierno debía articular alguna forma factible de socorrer a quienes andaban merodeando en busca de limosnas, transitando por las mismas calles y los mismos lugares que el resto de la sociedad.

Estos centros de internamiento, desempeñarian un papel a la vez de ayuda y de represión, porque su destino era socorrer a los pobres, pero era normal la existencia de celdas de detención e incluso alas enteras donde encerrar a los pensionados.

Al rol social atribuido como lugar de caridad, se le añade el papel de reformar a las personas que allí llegasen, normalmente con pautas de con-

Archivo Municipal de Santa Cruz de Tenerife (AMST). Cárceles. 1823, s/fol. En las Islas Canarias no tenemos constancia de que esto se llevase a cabo, debido sin duda a la frustración de ese periodo constitucional, con lo que esas medidas quedaron en proyectos que se retomarían con posterioridad a 1837 
ducta poco aceptadas por los bloques de poder. Las normativas internas debían ser bastante rígidas como cabe pensar por la perfecta separación interna, tanto en los hospitales como en los hospicios.

También tenemos testimonios de ordenamientos de reclusión de personas acusadas simplemente de holgazanería. Éstas debian ser internadas en los hospicios ${ }^{18}$. El no poseer un trabajo ha pasado a convertirse en un delito, la normativa prohíbe una situación totalmente involuntaria y que por sí sola ya supone una desgracia. Ambas concepciones aparecen en la documentación, en ocasiones entremezcladas, siendo difícil deslindar la una de la otra.

En el siglo xvIII se veía necesaria una modernización de las estructuras de la beneficencia que permitiese dar la coherencia requerida a los programas prácticos cuya implantación ya no admitian más demora.

Para ello era preciso realizar diversas innovaciones. Desde Felipe $V$ se había encargado especialmente a la Sala de Gobierno, y a la Comisión de hospitales del Consejo como principal protector de ellos, el exclusivo conocimiento de lo relativo a los mismos, a fin de ejercer su control. La politica centralizadora de la dinastía borbónica también abarcaba este sector.

Los hospitales durante la Edad Moderna bascularon entre el doble cometido, enfrentado dialécticamente, de ayuda a los necesitados y represión de los sectores más deprimidos de la población. Ambas dedicaciones estuvieron encaminadas a evitar posibles convulsiones sociales generalizadas, sobre todo en un periodo de fácil alteración del ciclo económico, que arroja a grupos cuantiosos de personas al desempleo y la marginalidad, entiéndase mendicidad, latrocinio, prostitución, etcétera.

Durante estos tres siglos, la cárcel existió, pero generalmente el mantenimiento de los presos debía ser sufragado por medio de la expropiación de los bienes de los prisioneros, puesto que los poderes públicos dedicaron escasos recursos económicos para las prisiones.

La cuestión planteada fue qué hacer con aquellos que, sin poseer absolutamente nada, andaban molestando con sus acosos y su presencia a las clases acomodadas. Las quejas eran reiteradas, principalmente a finales del siglo XVIII y primer tercio del XIX.

La alternativa ofertada por los tiempos modernos fue la reutilización de los hospitales que históricamente habian tenido tareas ambiguas, fluc- 
tuantes entre la sanidad y el refugio para los viajeros que andaban por las rutas de peregrinación.

Sin embargo, existían dos tipos de pobres: los de solemnidad y los vergonzantes. Los primeros eran los de siempre, aquellos que nunca antes ni después saldrian de esa situación, mientras que el segundo grupo designaba a aquellos que habían tenido una buena posición y habian quedado sin recursos económicos. Generalmente ambos grupos estaban separados. A los vergonzantes se les destinará una sala de los hospitales denominadas cuartos ocultos, que gozaban de mayor espacio y menos problemas de masificación, además de guardar el secreto de su identidad. Incluso, en alguna ocasión hallaremos en las providencias del Consejo la reglamentación de una institución dedicada exclusivamente a la asistencia de los pobres vergonzantes ${ }^{19}$.

El fenómeno general europeo del vagabundeo fue aumentando con las guerras y las epidemias. Hubo mayores facilidades de movilidad territorial en los tiempos modernos, por lo que también se produjo un incremento en la difusión de la opinión adversa a este hecho, que promovió en primer lugar la creación de hospitales, con distintas denominaciones. Objetivamente cumplian la misión de reunir a los grupos marginales que no tenían un lugar concreto dado su carácter errante ${ }^{20}$.

De esta forma va surgiendo a comienzos del Antiguo Régimen la idea de hospital, que predominaría a lo largo de más de tres siglos con diversas acepciones que partían de esos pensamientos. Al mismo tiempo, los conceptos de pobre, mendigo, vago, también irán evolucionando, lo que incidirá en las instituciones benéficas al alterarse los sujetos susceptibles de ser encerrados en ellas variando el resultado final.

Este sistema institucional de la Edad Moderna, encargado de mitigar el peso de la pobreza y el dolor de la enfermedad, se hallaba muy inspirado por la fuerte religiosidad imperante, en un momento de preocupación por el más allá y de cambio en la actitud ante la muerte, porque se trata de ganar la eternidad con las buenas obras de caridad, prestándose normalmente más atención a la salvación de las almas que a la de los cuerpos.

Biblioteca del Ministerio de Justicia. Providencias del Consejo, fols. 220 recto-225 recto Maraval. . J.A., "De la misericordia a la justicia social en la economía del trabajo: la obra de fray Juan de Robles". Moneda y Crédito, n." 148, Madrid, marzo-1979, pág. 83. Para el caso francés contamos con el libro HUFTON. O. The poor in Eighthteenth-Century France 1750-1789. Oxford, Claredon Press, 1974. 
La variedad de los centros asistenciales en funcionamiento fue muy grande. Habia hospitales para cada uno de los dos sexos, o para ambos al mismo tiempo; bien enfermos o bien pobres, o las dos cosas; para comunidades regionales, para extranjeros; para enfermedades contagiosas, especialmente encaminados a combatir "las bubas», afección de transmisión sexual; para enfermos incurables e impedidos; para locos; para leprosos; para convalecientes. Más aún, establecimientos que acogian a los niños expósitos o huérfanos; asilos para ancianos y viudas pobres; hospederías para viajeros y peregrinos; Casas de Misericordia y Albergues para pobres, sin olvidarnos de los reformatorios para jóvenes de ambos sexos y las casas de reforma para mujeres "arrepentidas".

De esta manera, el conjunto institucional hospitalario se completaba con una serie de establecimientos más marcadamente represivos, cuya finalidad principal era aislar a los elementos considerados antisociales y peligrosos para el resto de la sociedad ${ }^{21}$.

Según Soubeyroux, la importancia otorgada a las visitas y exámenes de los médicos en el último tercio del siglo XvIII traduce también una evo. lución característica en la imagen del hospital, que deja de ser un lugar de asistencia para convertirse en área de vigilancia en que el papel esencial se atribuye al médico ${ }^{22}$. Creemos que no es correcto valorar únicamente esta función, ya que una no excluye a la otra, la higiénica y sanitaria.

El Estado, entendido en su vertiente de instrumento de la clase dominante, no sólo tiene una función coercitiva, sino que ejerce una serie de atribuciones que no se reducen a la dominación política. Es en este plano donde debemos encuadrar la vertiente asistencial-sanitaria de las instituciones benéficas del Antiguo Régimen, aun admitiendo que forman una parcela del Estado. Pensar lo contrario es un simplismo mecanicista en el cual se ha caído en ocasiones, exponiéndose la represión y la ayuda a los hospitales como elementos disociados. Es decir, en nuestra opinión durante los Tiempos Modernos, la idea de hospital fue mucho más amplia y compleja que en nuestros días: eran establecimientos públicos que guardaban una estrecha conexión con el entorno social, por lo que las variaciones de éste condicionaban la marcha de aquél.

Carmona Garcia, J.I., "La Asistencia Social en la España de los Austrias". en Seminario de historia de la accion social. De la beneficencia al bienestar social. Madrid, Siglo Veintiuno, 1986. págs. 73-74.

Soubeyroux, J., “Sátira y utopía de la Corte en aventuras de Juan Luis de Rejón y Lucas (1781)". En Equipo Madrid: Carlos III. Madridy la llustración, Madrid, Siglo Veintiuno. 1988, pág. 409 . 
Durante el siglo XVIII en los hospitales era asistido el colectivo poblacional que no estaba integrado, ni desde el punto de vista familiar ni profesional, y que no contaba con medios materiales para ser atendidos a domicilio que era lo que hacían las personas pudientes. Pero lo cierto es que la realidad era mucho más compleja. Las instituciones benéficas forman parte de la plasmación práctica de una política cuya finalidad era enfrentarse a los problemas generados por desvalidos y vagabundos, entendiéndose el internamiento como la salida óptima para mantener las estructuras socio-económicas.

Al transcurrir el tiempo, los hospitales acumularon las funciones de una institución penal, asilo, taller y centro sanitario, confundiendo el resultado final tanto para nosotros como para su propia época.

La finalidad del internamiento como centro de corrección era la de mantener la autoridad paterna y el honor de la familia, proteger la religión católica, defender el poder real y preservar el orden y la seguridad pública ${ }^{23}$.

Sin embargo no debe descuidarse la actividad curativa de los hospitales, olvidada en algunos trabajos, aunque puesta especialmente de relieve en los estudios sobre historias médicas. En este sentido debemos destacar que uno de los rasgos que caracterizan la epidemiología de la segunda mitad del siglo XVIII es la frecuencia y gravedad con que las fiebres pútridas malignas, o fiebres tifoideas, afectaron a la población española y europea en general. En muchas ocasiones se alcanzaron cotas dramáticas en la extensión e intensidad de las afecciones. Esto motivó la utilización de los hospitales en las políticas sanitarias de determinadas coyunturas aunque lo más probable es que el objeto principal fuese aislar el virus entre esas paredes. Los trabajos recientes ponderan más esta finalidad que quizás posea mayor importancia a nivel histórico, porque tiene una incidencia directa en las estructuras socio-económicas que forman parte de la intrahistoria, como brota, a veces implícita y otras veces explícitamente en la documentación de la época.

Existian dos canales por los cuales ingresaba el recluso por mala conducta. En primer lugar, estaban aquellas personas que por vagabundeo, pequeños hurtos $u$ otras actitudes de esta clase, eran socialmente indeseables y enviados a estos centros por las autoridades con el objeto de mantener el orden. En segundo término, por ser menos numerosos, nos 
encontramos con algunos internados por sus propios familiares para que se enmendasen. En este segundo supuesto, eran considerados como voluntarios, por lo cual son puestos junto con aquellos que iban por su propio pie, resultando imposible que podamos cuantificar los diferentes grupos por falta de datos fiables.

La percepción del vagabundo también atenta contra la estética de las ciudades. No es bueno para mantener la armonia social las visiones de impedidos implorando ayuda en las plazas e iglesias además del lógico trastorno que suponía para los sectores acomodados y la sensación de caos con que impregnaba al paisaje urbano.

La beneficencia pasó a utilizarse como un poderoso medio de control de un grupo de población que vivía en la miseria aceptando su papel por ser conscientes de que podían subsistir por medio de la caridad, atenuándose de este modo las contradicciones sociales, al no quedar al borde de la muerte, sobre todo aquellos que ponian en práctica todo tipo de picaresca para vivir.

En la Novísima Recopilación se recogía una Cédula en la que se plasma el pensamiento del Papado ante el tema:

"Cuando no se fundaren o erigieren tales recogimientos, o no conviniere colocar o recluir en los erigidos a todos los pobres, será el objeto, según el Breve, establecer y promover por otros medios el consuelo, socorro y remedio de las necesidades; desterrando y evitando, como su Santidad encarga y desea, la codicia de aquellos que pasan la vida en el ocio y mendiguez voluntaria, en perjuicio de los verdaderos pobres, cuyas limosnas defraudan" ${ }^{24}$.

Otro aspecto que debemos señalar entre las funciones de las instituciones benéficas, es el ideológico. En estos establecimientos se pretendía regenerar a los recluidos de quienes se suponía una conducta poco ejemplar. Se trataba por todos los medios de que confesasen y comulgasen, antes incluso de recibir la visita del médico. Esto estaría relacionado con la férrea disciplina existente en el interior y principalmente con la obligada participación diaria en los oficios religioso para encauzar en la doctrina cristiana a los internos.

Es decir, que existía de forma genérica una pluralidad de funciones, abarcando desde la recogida y asilo de pobres hasta la atención de niños huérfanos o abandonados. Aunque, como indicábamos, podemos observar

2. Novisima Recopilación de las leyes de España, Libro I, Titulo XXV, Del Fondo pio beneficial, Ley I, Cédula, 1-XII-1783. 
una cierta especialización en lo referente a la actividad desarrollada, por las enfermedades a las que se dedican o por la procedencia o calidad de las personas ingresadas en sus dependencias.

Juan Luis Vives en el primer tercio del siglo XVI denominaba hospitales a las casas en las cuales eran alimentados y cuidados los enfermos, se educaban jóvenes de ambos sexos, se criaban niños expósitos, eran encerrados los locos y pasaban su vida los ciegos. Refleja esa multiplicidad de grupos para los cuales se destinaban esos organismos señalando como responsables del tema a los gobernantes de las ciudades.

El humanista valenciano apuntaba seis características que debian cumplir las instituciones benéficas para que fuesen de utilidad a la sociedad. Las expondremos de forma muy sintética a continuación, ya que en estas consideraciones se asentó gran parte del pensamiento moderno sobre el tema, en primer lugar en el noroeste de Europa y posteriormente en el área mediterránea:

1. Será un honor para la ciudad hacer desaparecer a los mendigos y con ello obtener una mayor atención del bien público.

2. Se producirán menos delitos contra la propiedad privada, porque se mitigará y disminuirá la necesidad, que es la principal causa de estas malas costumbres.

3. Estando todos provistos, habrá mayor quietud en el público.

4. Será más segura y agradable la asistencia a los templos, así como andar por la ciudad en general, evitando ver escenas desagradables que horrorizan a la naturaleza y especialmente al ánimo humano.

5. Los menos acomodados no se verán forzados a dar limosnas sólo por la importunidad, y si alguno quisiera ofrecer algo no se cohibirá por ser muchos los mendigos ni por el recelo a dárselo a una persona indigna.

6. La ciudad ganará al tener vecinos más modestos, cívicos, bien criados y sociables, ya que se sustentan en ella, no pensando en novedades alteradoras, sediciones o tumultos ${ }^{25}$.

Como puede observarse, el colofón señala el papel que deben cumplir los hospitales, no en una línea represiva, sino atenuando las contradicciones al proporcionar medios de vida al conjunto de la población.

VIVFS, J.L., Tratado del Socorro de los pobres. Valencia, Imprenta de Benito Monfort, 1781 págs. $239-241$ 
Según la opinión de los autores de la época, fueron los hospitales, en cuanto lugares destinados al recogimiento y cuidado de pobres y enfermos, las instituciones que mejor respondian a la organización de la beneficencia en el Antiguo Régimen. En manos de la Iglesia y de los particulares, de las cofradías o de las autoridades locales, estos centros estaban asumiendo desde varias centurias atrás, el protagonismo en la asistencia al necesitado, siguiendo unos moldes tradicionales que tenían su fundamentación teórica en las Sagradas Escrituras y en el derecho del pobre a recibir limosnas ${ }^{26}$.

En varias Reales Ordenes del siglo XVIII se manda el internamiento de mujeres, niños e impedidos en casa de misericordia, en donde se les debía tratar con "misericordia", al tiempo que los aplicaban al trabajo o les enseñaban, a los más capacitados, según su edad y fuerzas, para que pudiesen reincorporarse en un momento dado a la vida económica.

Carlos III disponía con el mayor celo y cuidado el recogimiento de las personas imposibilitadas para desarrollar algún tipo de actividad productiva, pero con esto no solventaba el problema de los enfermos temporales o de los parados a consecuencia de la adversa coyuntura económica 0 de las madres solteras o viudas. Estos grupos no podian ser considerados como mendigos, pero era preciso arbitrar medidas para ellos, y efectivamente durante estos años se llevaron a la práctica multitud de iniciativas para atender a todos estos colectivos. Los distintos organismos gestores de esa política hicieron extensivo su cometido hacia aquellos que en algún momento fuesen declarados pobres de solemnidad o pobres vergonzantes.

Así, la ley promulgada el 26 de agosto de 1785 disponía que fuesen recogidos los mendigos deformes o lacerados, tanto para proceder a su curación, cuanto para evitar al público los sentimientos que producian siempre, al estar a la vista, sus llagas y demás señales que salian en la piel ${ }^{27}$.

Sobre los establecimientos de locos se tiene conocimiento para diversas regiones de la Monarquía. Es significativo el cuadro de Goya "Casa de locos" en el cual se percibe la atmósfera en que vivian, con un duro régimen disciplinario ya que incluso pinta guardianes con el látigo en la mano. El hecho de haberse inspirado en ello es indicativo de que no era

Marcos Martin, A., Economia, sociedad, pobreza en Castilla: Palencia, 1500-1814. Palencia, Diputación Provincial de Palencia. 1985, t. II. pág. 450.

DE NENClaRes, E.M., Legislación española de Beneficencia. Madrid, Librería de Don León Pablo Villaverde, 1859 , pág. 35 
algo extraño, puesto que sabemos que por su estilo costumbrista buscaba los modelos, objeto de la obra, en la vida cotidiana.

Al loco se le trata de internar para proteger a la sociedad de sus actos, potencialmente peligrosos. La situación tardó bastante en cambiar, porque la idea que se tenía de los locos no podía transformarse rápidamente, y era preciso seguir defendiéndose de él puesto que atentaba frontalmente contra el orden establecido y las normas de convivencia.

Los estudios realizados para el noroeste de Europa demuestran que la asistencia pública se dedicaba fundamentalmente a encerrar a sus asistidos por locura, libertinaje o alcoholismo. En una tabla elaborada por Diederiks y Spierenburg sobre las causas más frecuentes de internamiento se señalan de mayor a menor: el alcoholismo, la locura, el comportamiento licencioso en general, las amenazas normalmente relacionadas con malos tratos, las tendencias suicidas, la dilapidación del dinero, el vagabundeo, las trabas a la acción de la justicia y finalmente un pequeño grupo de conductas que aparecen mal definidas en la documentación ${ }^{28}$.

Es de enorme interés esta lista, que coincidia en todos los centros de este tipo, aunque para nuestro ámbito de estudio resulta absolutamente imposible conocer con exactitud las causas de los internamientos, porque nunca se especifican las razones de las mismas.

El tema de la recogida de mujeres también es de gran importancia, ya que, por ejemplo, en la mayor parte de las instituciones benéficas canarias, existia un claro predominio de este sexo. Es decir, que otra función seria la acogida de mujeres por diversos motivos. Debemos tener en cuenta que su situación jurídica en la sociedad del Antiguo Régimen era más desprotegida que la de los varones y en los momentos de crisis, la supervivencia de las mujeres en actividades marginales era más difícil.

Así, en el archipiélago canario ${ }^{29}$ el nivel de prostitución era elevado, por lo que habia varios hospitales que por sus primitivas ordenanzas debian ocuparse en recoger y curar a quienes padecieran enfermedades de transmisión sexual y, además, una Casa de Mujeres Arrepentidas en Las Palmas.

Pero habia muchas más razones por las cuales llegaban las mujeres a estos establecimientos. Así, en algunas residencias se reservaba alguna

\footnotetext{
DIEDeRIKS, H. y SPIERENBUhG, P., "L'entermement non criminel en Hollande, XVIllemeXIXème siècles" en Jacques G. Petit: La prison, le bagne et lihistoire, médecine et hygiène. Suiza, 1984, págs. 49-50.

Monzon Perdomo, M.E.. Marginalidad social en Canarias durante el siglo xvil.. La Laguna Caja Canarias-Universidad de La Laguna, 1990 (microfichas)
} 
sala destinada a recoger a aquellas mujeres que habian quedado embarazadas sin tener el cobijo familiar, grave deshonor que dificultaba las posibilidades de subsistencia de las madres y sus hijos.

En el artículo 44 de la Ley General de Beneficencia Pública se puntualizaba que era preciso tener la conveniente separación entre las mujeres acogidas, según sus distintas circunstancias y la conducta pública que hubiesen observado. Con esto se admite por vía legal la diversidad de cauces que ponen en la marginalidad a muchas mujeres, que luego son objeto de internamiento, con notables diferencias entre unas y otras.

En el artículo 41 de la ley mencionada se señalaba asimismo que en cada provincia debia haber una casa de maternidad con tres departamentos: uno de refugio para las mujeres embarazadas y recién paridas, otro para la lactancia de los niños y, finalmente, otro para conservar y educar a estos hasta la edad de seis años ${ }^{30}$. Esta especialización de los establecimientos supone una visión avanzada con respecto a la cuestión. En la Cuna de Expósitos de Las Palmas, sabemos que con año y medio ya se consideraba que los niños habían acabado la fase de amamantamiento, por lo que las amas los devolvían al torno, quedando al cuidado de la institución hasta que una persona los adoptase ${ }^{31}$.

Hasta fines del siglo XVIII existió una elevada mortalidad en las instituciones benéficas, hasta el extremo de incidir en el porcentaje global de fallecimientos de las ciudades, según se desprende de algunos estudios demográficos. Con este dato se pone aún más en tela de juicio el papel de los establecimientos de recogimiento, porque eran incapaces de asegurar la vida de los indigentes, que no estarian en peor situación sanitaria fuera que dentro, constituyendo un elemento de disociación entre lo pregonado por las autoridades y la trágica realidad.

Durante el Antiguo Régimen, los objetivos que debian cumplir las instituciones benéficas (al menos sobre el papel), se vinculaban también estrechamente con la dernanda u oferta de mano de obra en las distintas coyunturas económicas. Surge así una política social que, sobre el único fundamento de la aptitud para el trabajo, establecía una discriminación entre el pobre inocente (es decir, el anciano, el niño, el inválido, la mujer desamparada) y, por otro lado, el pobre culpable, es decir, el joven y el hombre maduro desocupados por su vagancia. A las necesidades de supervivencia

Archivo Municipal de La Laguna, Fondo del Antiguo Cabildo de Tenerife. Sección segunda sig: R-IV. Reales Órdenes e Instrucciones, número 3, 1822-1828, fol. 12

Archivo Diocesano de Las Palmas. Sin catalogar. Hospital de San Martin. s/fol. 
del primer grupo, se intentará hacer frente a través de la organización asistencial; para el segundo grupo se utilizará la entrada coactiva en el conjunto de las instituciones surgidas en Europa en los siglos XVII y XVIII.

Estos establecimientos asistenciales debian introducir la disciplina y la ética manufacturera entre quienes eran, por origen y educación, extraños a la colectividad dominante ${ }^{32}$, es decir, trataban de hacer extensiva a toda la población la nueva filosofía sobre el trabajo.

En el continente europeo surgieron en relativamente poco tiempo establecimientos de estas características, con diversas denominaciones: tuchthuizen, hôpitaux généraux, workhouses y Zuchtäusern.

Estas instituciones eran en parte lugares de producción artesanal concentrada y su finalidad principal debía ser la de separar a todo aquel que pudiese tener un comportamiento poco adecuado. Era preciso disciplinarles mediante un estricto régimen de trabajo, inculcarles principios morales y convertirles en mano de obra dócil y productiva, aunque es evidente que resultaba imposible recluir a todos los parados en estas casas.

Por lo que se desprende de los textos, podemos pensar que la mayor parte de los pobres que quedaban en libertad estuvieran aterrorizados por la perspectiva de verse atrapados en esos lugares y que por tanto pudieron estar dispuestos a trabajar para conseguir su manutención diaria. Al menos eso trataban de conseguir algunos dirigentes, pero también debemos tener presente que los hospicios pretendian antes castigar y disciplinar a los mendigos y vagos que darles un trabajo digno con el cual poder vivir ${ }^{33}$.

Había una serie de trabajos para los cuales se necesitaba mano de obra, a ser posible bastante barata, bien obras públicas, bien nuevas actividades que comenzaban a implantarse en esos momentos. De esta forma, estaba previsto que los pobres mendigos, válidos e inválidos de uno $u$ otro sexo, fuesen encerrados en un hospital u hospicio para ser empleados en obras, manufacturas y otros trabajos ${ }^{34}$.

La segunda mitad del siglo xvill constituye una nueva etapa de aceleración de las iniciativas en el campo asistencial, singularizada por la necesidad de hacer frente a los problemas derivados del crecimiento econó-

\footnotetext{
Pavarint. M.. Control y dominación. México, Siglo Veintiuno, 1983, págs. 32-33.

LIS. C. y Soiy. H.. Pobreza y capitalismo en la Europa preindustrial (1350-1850). Madrid. Akal, 1985. pags 138-139.

DH pauw. J.. «Pauvres, pauvres mendiants, mendiants valides ou vagabonds? Les hésitations de la législation royale". Revue d'histoire modeme et contemporaine. Paris, julio-septiembre 1974, pág. 403.
} 
mico y de la rápida expansión demográfica de las ciudades, por la respuesta relativamente apresurada a las situaciones críticas y por el esfuerzo de racionalización y generalización de las medidas emprendidas bajo la dirección de las autoridades públicas.

La primera diferencia, y la que resulta más evidente, es la que podemos observar entre los grandes establecimientos benéficos encargados de acoger a una población más o menos numerosa de internos y que de alguna manera dan la pauta en este campo, y las instituciones que ejercen una asistencia externa, en forma de raciones alimenticias, entregas de ayudas económicas $u$ otras formas de ejercer caridad ${ }^{35}$.

Los adultos que por su edad o educación fuesen incapaces de aprender los elementos de las artes y oficios, serian destinados a los otros ejercicios más groseros para los que solamente se necesitaba fuerza. Los ancianos que pudiesen, habian de servir para recoger las limosnas para el instituto, trabajando en algunos oficios internos en las casas-hospicios, dedicándose a su aseo y limpieza juntamente con la de los niños reclusos, sirviendo de pedagogos los más capacitados, llevando y trayendo al centro a aquellos jóvenes que desarrollasen su actividad fuera, impidiendo que fuesen solos para que no se quedasen con los otros muchachos del pueblo, por la intoxicación moral que ello supondría, puesto que pensaban que aquéllos eran de malas costumbres. Las ancianas que aún fuesen hábiles podrían emplearse en labores de aseo de las casas y vigilancia de las niñas más pequeñas.

Una Real resolución de! 21 de julio de 1780 introducía otra variante por la cual se integraba al huérfano desde los pocos años al mundo laboral. Los jóvenes aptos por su robustez para trabajar en la agricultura - sector que se desea potenciar en estos momentos-, una vez adquiridos los conocimientos básicos de la doctrina cristiana y enseñanza de primeras letras, se entregarian a un labrador acomodado para que los educase y se sirviera de ellos como mano de obra en sus propiedades ${ }^{36}$. Era una especie de relación contractual que proporcionaba brazos para los campos a muy bajo precio, al tiempo que suponía un ahorro considerable en materia de beneficencia al deshacerse de un grupo de bocas a las que era necesario alimentar.

A estas indicaciones que hemos hecho, debemos añadir que efectivamente la proporción de los necesitados, en casi todos los lugares, estaba

MaRIINEz SHAw, C.. "L'assistència pública a la Barcelona Moderna". L'Avenç: Revista d'história, n’ 91, Barcelona, marzo-1986, págs. 32-33

sh. Novisima Recopilación de las leyes de España. Libro VII, Titulo XXXVIil. De los hospitales, hospicios y oiras casas de misericordia, Ley $V$. 
formada por párvulos, viudas y ancianos, en condiciones físicas desfavorables, enfermos o débiles, personas que quizás por su incapacidad natural no podian ganar el salario minimo de un adulto en buenas condiciones para vivir de su fuerza de trabajo o que tal vez estaban impedidos para realizar cualquier tipo de actividad laboral.

A pesar de todo, ninguna de estas instituciones tenía posibilidades reales de erradicar la marginalidad con la incorporación de los indigentes a la vida laboral. Incluso algunos hospitales que cumplian una función asistencial fomentaban la mendicidad, ya que era una forma de obtener recursos, mientras las casas donde se les obligaba a trabajar resultaban tan odiosas que incitaban a los pobres a mantener su libertad por todos los medios posibles.

Para Cataluña, Monserrat Carbonell ha demostrado que al mismo tiempo las instituciones benéficas constituian un sistema de regulación y control del mercado laboral, asegurando la supervivencia de un ejército industrial de reserva, compuesto por mujeres, hombres y jóvenes que, mediante una disciplina estricta, se convertian en fuerza de trabajo dócil y útil ${ }^{37}$.

Podemos concluir con Christine Buci-Glucksmann diciendo que en sentido restringido, el Estado se identifica con el gobierno, con el aparato de la dictadura de clase, en tanto debe cumplir funciones coercitivas y económicas. La dominación de clase se ejerce por medio del aparato del Estado en sentido clásico, donde se encuadran los centros benéficos. Pero esta función coercitiva a la que aludimos es inseparable de un cierto papel adaptativo-educativo del Estado, que busca realizar una adecuación entre el aparato productivo por un lado y por otro la moralidad de las masas populares ${ }^{38}$.

Además del adoctrinamiento cristiano y las enseñanzas de un oficio, estas instituciones benéficas conjugan otro papel doble. Por un lado, actúan como sustitutivos de la familia, para los huérfanos, los enfermos, los viejos, etc.; y, por otra parte, como defensores de la reputación pública de algunas familias al proporcionarles lugares en los cuales podía ocultarse el escándalo, en particular el de naturaleza sexual ${ }^{39}$.

Carbonell. I Estel.lef, M., "La beneficencia a finals del s. XVIIl: una aproximacio. La casa i Hospital de Misericordia de Barcelona". Primer Congres d'Historia Moderna de Catalunya. Barcelona, 1984, t. I, pág. 794. Posteriormente la misma autora ha desarrollado este planteamiento en su tesis doctoral: Fobresa i estratègies de supervivencia a Barcelona a la segona meitat del s. XVIII. Les acollides a la Casa de Misericordia i el Mont de Pietat de Nira. Sra. de I'Esperança, Universidad Autónoma de Barcelona, diciembre de 1992

Buci-Glucksmann, C., Gramsci y el Estado. Madrid, Siglo Veintiuno, 1978, pág. 122.

Wool.t. S. Los pobres en la Europa Moderna. Barcelona, Crítica, 1989, pág. 44. 